\title{
Editorial
}

\section{Mathematical Methods for Heat Transfer and Thermodynamic Analysis of Conductive, Convective, and Radiative Media}

\author{
Mohsen Torabi, ${ }^{1}$ Nader Karimi, ${ }^{2}$ and Shohel Mahmud ${ }^{3}$ \\ ${ }^{1}$ Department of Mechanical and Biomedical Engineering, City University of Hong Kong, 83 Tat Chee Avenue, Kowloon, Hong Kong \\ ${ }^{2}$ School of Engineering, University of Glasgow, Glasgow G12 8QQ, UK \\ ${ }^{3}$ School of Engineering, University of Guelph, Guelph, ON, Canada N1G 2 W1 \\ Correspondence should be addressed to Mohsen Torabi; mohsen.torabi@my.cityu.edu.hk
}

Received 17 January 2016; Accepted 17 January 2016

Copyright (C) 2016 Mohsen Torabi et al. This is an open access article distributed under the Creative Commons Attribution License, which permits unrestricted use, distribution, and reproduction in any medium, provided the original work is properly cited.

The rapidly growing needs for energy and their associated environmental problems have led to the formation of most challenging issue facing human civilization. This has massively signified the role of energy analysis and optimization in a wide range of engineering disciplines. Further, the recent advancement in manufacturing of small scale devices and introduction of synthetic materials has added new dimensions to energy analysis. Historically, thermal energy analysis included first law investigations or heat transfer analyses. Over the last two decades, it was demonstrated that such analyses could be lacking and do not provide a complete picture for many applications. Hence, combined first and second law analyses were conducted mostly on convective systems. More recently, the problems which chiefly involve conduction in complex media, such as porous or multilayer media, started to attract attention of the research community. This class of problems is essential in a number of applications including energy systems, energy storage, underground reservoirs, and micro- and nanoscale manufacturing. Optimization of combined conduction, convection, and radiation of heat and the resultant generation of entropy in these applications introduces a very rich and mostly unexplored problem.

This special issue brings about various problems in these fields through comprehensive considerations. Editors hope that provided problems and investigations help engineers and scientists regarding optimum thermophysical designing conditions for discussed systems.

Mohsen Torabi

Nader Karimi

Shohel Mahmud 


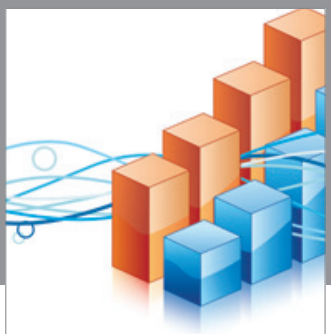

Advances in

Operations Research

vatem alat4

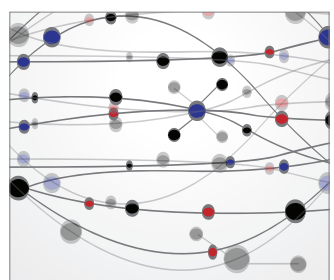

\section{The Scientific} World Journal
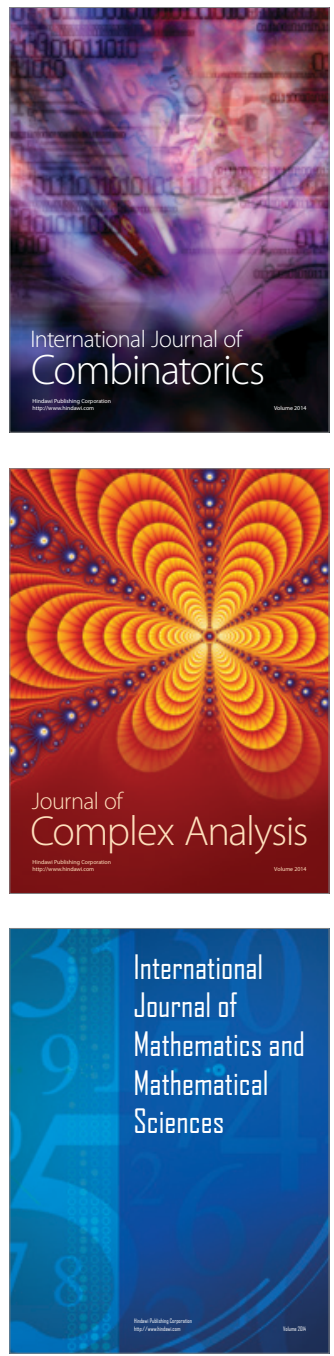
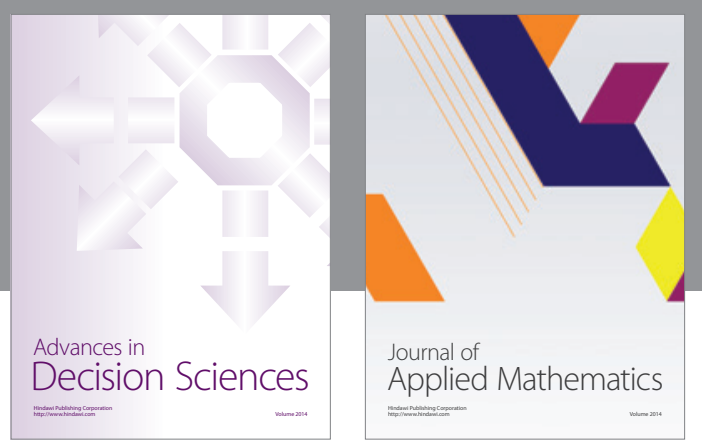

Algebra

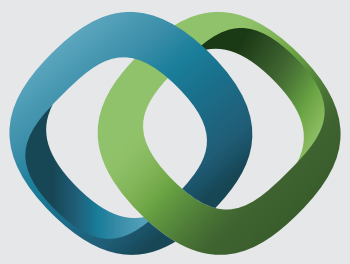

\section{Hindawi}

Submit your manuscripts at

http://www.hindawi.com
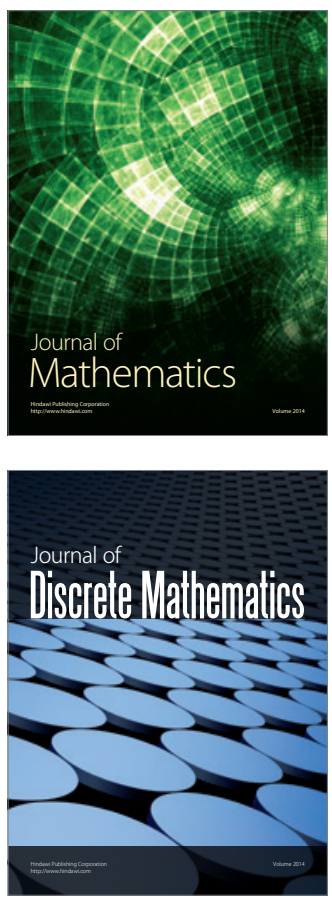

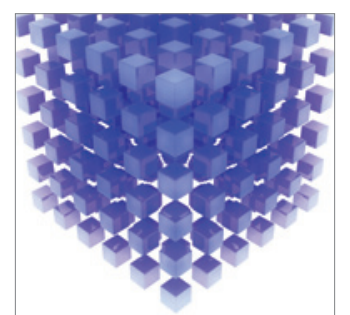

Mathematical Problems in Engineering
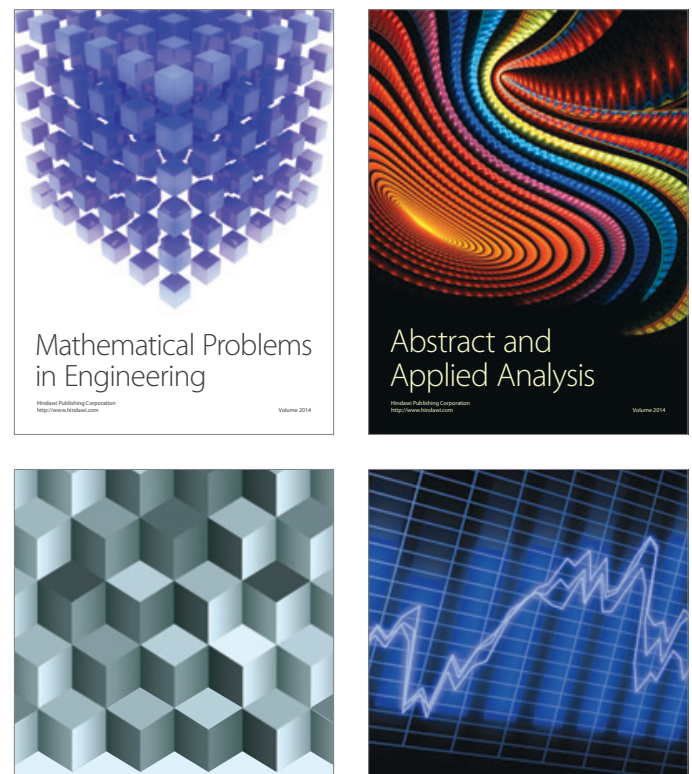

Journal of

Function Spaces

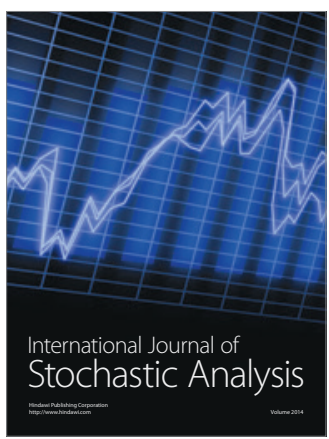

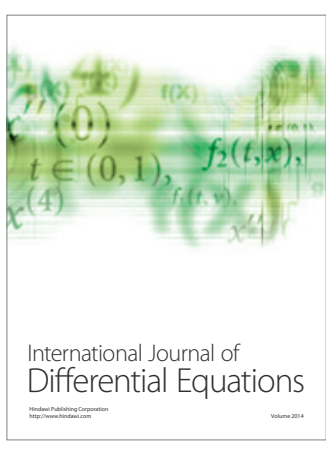
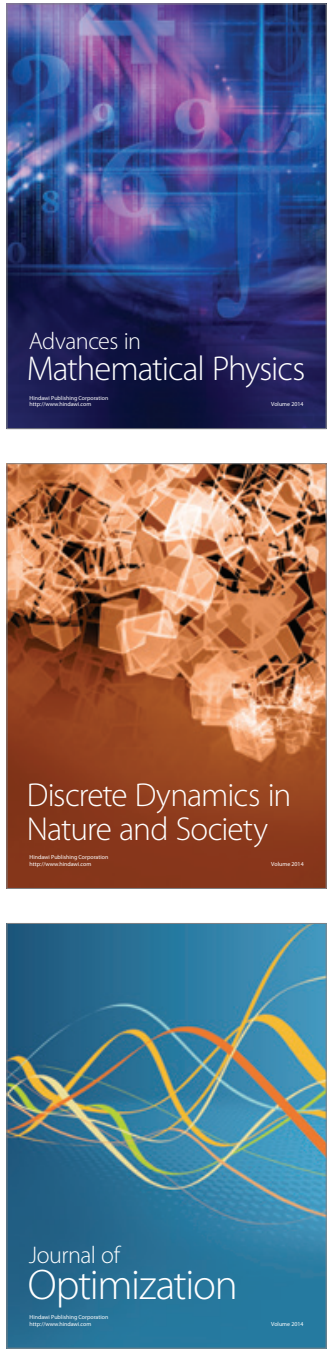